\title{
ANÁLISIS DE LA CULTURA ORGANIZACIONAL DE LAS EMPRESAS DE SERVICIOS DE LIMA METROPOLITANA
}

\author{
ANALYSIS OF THE ORGANIZATIONAL CULTURE OF SERVICE COMPANIES \\ IN METROPOLITAN LIMA
}

\author{
${ }^{1}$ Ampelio Ferrando Perea
}

\begin{abstract}
Resumen
La presente investigación tuvo como objetivo analizar la eficacia de la cultura organizacional de las empresas de servicios de Lima metropolitana, proponiendo acciones de mejora de algunos factores de la cultura organizacional. El estudio describe y evalúa la eficacia de la cultura organizacional en base a cinco variables: comunicación, trabajo en equipo, desarrollo personal, adaptación al cambio y mejora de la calidad y productividad, fundamentalmente mediante la aplicación de un cuestionario de 22 reactivos a 300 colaboradores de 25 empresas de servicios. El resultado final manifiesta en estas empresas una eficacia de la cultura organizacional del 67.8\%, que como conjunto de valores, tradiciones, creencias, hábitos, normas y actitudes interiorizadas contribuye efectivamente al logro de sus objetivos económicos y sociales.
\end{abstract}

Palabras clave: cultura organizacional, eficacia, comunicación, trabajo en equipo, desarrollo personal, adaptación al cambio, mejora de la calidad y productividad.

\begin{abstract}
The present research had as objective to analyze the effectiveness of the organizational culture of service companies in metropolitan Lima, proposing actions to improve some factors of organizational culture. The study describes and evaluates the effectiveness of organizational culture based on five variables: communication, teamwork, personal development, adaptation to change and improve quality and productivity, primarily through the use of a questionnaire of 22 items to 300 employees belonging to 25 service firms. The end result manifests in these businesses an effective organizational culture of $67.8 \%$, which as a set of values, traditions, beliefs, habits, internalized norms and attitudes contributes effectively to achieving economic and social objectives.
\end{abstract}

Key words: organizational culture, effectiveness, communication, teamwork, personal development, change adaptation, improve quality and productivity.

\section{Introducción}

En la realidad del comportamiento organizacional en las empresas, para el caso del presente estudio de aquellas que prestan servicios, se hace necesario sondear cómo se ha venido desarrollando su cultura organizacional a través del desarrollo de sus actividades así como la gerencia ha incidido en que ella sea entendida, manejada por sus colaboradores, de tal manera que favorezca su desempeño. La cultura organizacional modela, guía, determina el comportamiento de los componentes del grupo organizado, sirviendo de marco de referencia a los miembros de la organización y dictando las condiciones acerca de cómo sus miembros deben conducirse en ésta. Esto es poco aparente en organizaciones pequeñas pero cada una de ellas desarrolla una cultura propia, particular y diferenciada, la cual se afianza con el transcurso del tiempo.

Según Aguilar (2009), el estudio de la cultura de una organización ha sido, históricamente, una tarea que sin duda reviste dificultades no sólo en el plano metodológico, sino también conceptual y epistemológico. El avance tecnológico y la globalización de la economía, son hoy los rasgos distintivos del mundo del trabajo organizado. En este contexto, una preocupación fundamental en la que parecen centrase los objetivos prioritarios de las organizaciones, tanto públicas como privadas, ha sido desarrollar dos aspectos a partir de la cultura.

De acuerdo con Schein (1992) la cultura es: "un modelo de presunciones básicas - inventadas, descubiertas o desarrolladas por un grupo dado al ir aprendiendo a enfrentarse con sus problemas de adaptación externa e integración interna, que hayan ejercido la suficiente influencia como para ser considerados válidos y en consecuencia ser enseñado a los nuevos miembros como el modo correcto de percibir, pensar y sentir esos problemas".

Pero, ¿Por qué se debe conocer de la cultura organizacional? ¿En qué beneficia a una organización? Cruz (2005), señala que los beneficios fundamentales de la cultura organizacional son: 
- Lograr un cambio más duradero en la organización.

- Crear la necesidad de mejorar la organización.

- Facilitar los cambios de métodos y estilos por medio del autoconocimiento.

- Crear la necesidad de modificaciones conductuales y adquisición de nuevas habilidades.

- Lograr mayor control de los recursos y organización integral.

- Movilizar los recursos humanos en la identificación de problemas y búsqueda de soluciones creativas.

- Mejorar el trabajo individual y grupal.

- Crear capacidad de autodiagnóstico y auto-diseño de la estrategia empresarial.

Por consiguiente, toda empresa, y entre ellas las de servicios, debe contar con una buena cultura organizacional de tal forma que su recurso humano se identifique con ella, les recuerde lo importante, relevante que es el mantener un buen comportamiento organizacional gracias a esa cultura organizacional que se ha venido dando en donde se manifiestan los resultados, logros, alcance y que le ha permitido salir adelante en el entorno donde opera. Sin embargo, son muchas las empresas que han fracasado porque no contaron con una sólida y auténtica cultura organizacional, propia de los tiempos modernos, así como prevención a los riesgos; han descuidado las ventajas que representa contar con una buena cultura organizacional, mucho más si esta es un auténtico producto del desarrollo de las actividades que viene realizado. Por ejemplo, según Fernández (2010), en México desconocer la nueva cultura organizacional es una de las áreas vulnerables de las empresas, siendo algunos de los problemas más comunes:

- La falta de conocimiento en la nueva cultura de desarrollo organizacional.

- Falta de claridad de cómo llevar sus producto o servicios al consumidor final.

- Incapacidad para fortalecer el área comercial y de ventas de la compañía.

- No conocer si el personal que tienen actualmente es el adecuado en un proceso de crecimiento.

- Resistencia al cambio.

La investigación de la cultura organizacional se ha desarrollado principalmente en los países altamente industrializados. En el Perú, los estudios empíricos realizados son escasos, y se han efectuado siguiendo muy de cerca los esquemas conceptuales y metodológicos norteamericanos.

En tal sentido, la gerencia peruana moderna no puede eludir lo que representa la cultura organizacional en pro del éxito de una empresa, todo lo contrario debe estar atenta como esta se desempeña, si se desea mejorar la competitividad y subsistencia de las empresas de servicios.

El objetivo general de este trabajo es evaluar la eficacia de la cultura organizacional de las empresas de servicios de Lima metropolitana, proponiendo acciones de mejora de algunos rasgos de la cultura organizacional. Este objetivo general se desglosa en los siguientes objetivos específicos: a) Medir la eficacia de la cultura organizacional de las empresas de servicios de Lima; b) Identificar ineficacias presentes en la cultura organizacional de las empresas de servicios y c) Proponer acciones de mejora de la cultura organizacional en las empresas de servicios.

\section{Materiales y métodos}

\section{Lugar de la investigación.}

La presente investigación se realizó en el ámbito geográfico de Lima metropolitana

\section{Tipo de la investigación.}

La presente investigación ha seguido las pautas de tipo descriptivo,pues describey evalúalacultura organizacional en función de cinco variables: comunicación, trabajo en equipo, desarrollo personal, adaptación al cambio y mejora de la calidad y productividad. Asimismo, es observacional, puesto que, los diversos fenómenos o acontecimientos han sido observados de tal manera que permiten un análisis e interpretación real de los hechos.

\section{Diseño de la investigación}

La presente investigación es de diseño no experimental seccional transversal, pues el cuestionario de la encuesta se aplicó en una sola ocasión, en un solo momento a los sujetos de estudio.

\section{Población y muestra}

Se determinó de forma aleatoria a 300 colaboradores seleccionados entre aquellos que pertenecen a 25 empresas del sector servicios, que decidieron colaborar en la realización del estudio.

\section{Técnicas de recolección de datos}

Dada las características, propiedades y atributos de las variables consideradas en la investigación; las técnicas empleadas para la obtención de los datos requeridos fueron: análisis documental, encuesta y observación directa.

Por cuestiones de la dinámica de las organizaciones y el tiempo disponible de los empleados de las organizaciones en estudio, se evalúa la eficacia de la cultura organizacional de las empresas de servicios de Lima metropolitana fundamentalmente a partir de la aplicación del cuestionario, evaluando las cinco variables establecidas. El modelo de cuestionario de 22 reactivos fue realizado en base al empleado por la Maestra en 
Psicología María Luz Pérez Lorenzo, profesora de la Universidad Veracruzana de México. (Andrade et.al., 2005).

\section{Procedimiento de análisis de datos.}

En una etapa inicial, se preparó una Carta de Presentación y un Proyecto de Investigación con el fin de solicitar la colaboración de diversas empresas de servicios, dando a conocer los aspectos más importantes inherentes al estudio. Con las organizaciones que respondieron positivamente a la propuesta de colaboración, se acordó la forma de administrar los cuestionarios y realizar actividades de observación directa.

Una vez recogida la información se procedió a su respectiva tabulación procesamiento, ordenamiento $\mathrm{y}$ análisis de los datos que se presentan en los resultados.

\section{Limitaciones del estudio.}

Este trabajo de investigación presenta varias limitaciones:

a) La primera limitación es el lugar, se realizó únicamente en el área geográfica de Lima metropolitana.

b) Otra limitación fue la decisión de los gerentes de algunas empresas visitadas de no permitir, debido a políticas de seguridad y ejecución de programas operativos, la realización de la encuesta a sus trabajadores. Tal situación condicionó la realización de la encuesta a los colaboradores de las empresas en las cuales sus gerentes gentilmente decidieron colaborar con la realización del estudio.

c) Otro factor limitante importante ha sido la disponibilidad de recursos financieros para la investigación, cubiertos por los fondos asignados por el Fondo Especial de Desarrollo Universitario- FEDU, fondo universitario de desarrollo de la investigación, de la Universidad Nacional Agraria La Molina.

\section{Resultados y discusión}

\section{Comunicación}

En la tabla 1 se aprecia que, los encuestados en las empresas estudiadas manifiestan en un $45 \%$ que se habla abiertamente para la resolución de problemas y en un $55 \%$ que no se practica una comunicación abierta con otros colaboradores y el personal directivo. También manifiestan en un $75 \%$ que la comunicación que se practica en las empresas es en ambos sentidos: de arriba hacia abajo y de abajo hacia arriba y un $25 \%$ que no se practica la comunicación de abajo hacia arriba, donde el personal solo recibe información y órdenes de sus jefes inmediatos. El 65\% afirma que los directivos manejan un estilo participativo para tomar decisiones importantes y por el contrario un 35\% manifiestan que rara vez comparten espacios para tomar decisiones con los trabajadores.
Tabla 1. Comunicación

\begin{tabular}{|c|c|c|}
\hline & Resu & tado \\
\hline Preg & Q & $\%$ \\
\hline 1) Comunicación abierta & & \\
\hline $\begin{array}{l}\text { a) Se habla abiertamente para resolver } \\
\text { problemas }\end{array}$ & 135 & 45 \\
\hline $\begin{array}{l}\text { b) No se habla abiertamente para resolver } \\
\text { problemas }\end{array}$ & 165 & 55 \\
\hline Total & 300 & 100 \\
\hline 2) Comunicación hacia arriba y hacia abajo & & \\
\hline $\begin{array}{l}\text { a) La comunicación hacia arriba es } \\
\text { tan importante como la de jefes a } \\
\text { subordinados. }\end{array}$ & 225 & 75 \\
\hline $\begin{array}{l}\text { b) La comunicación hacia arriba no es } \\
\text { tan importante como la de jefes a } \\
\text { subordinados. }\end{array}$ & 75 & 25 \\
\hline Total & 30 & \\
\hline
\end{tabular}

3) Espacio de toma de decisiones

a) En mi lugar de trabajo los directivos siempre comparten espacios para tomar 19565 decisiones importantes.

b) En mi lugar de trabajo los directivos rara vez comparten los espacios para tomar $105 \quad 35$ decisiones importantes

Total

Fuente: Elaboración propia.

$300 \quad 100$

\section{Trabajo en equipo}

Las empresas circunstancialmente recurren a todos sus empleados para resolver problemas. En la tabla 2 anterior se aprecia que el $63 \%$ de los directivos recurren a los empleados para resolver problemas, en tanto el 37\% afirma que en algunas ocasiones se toma en cuenta solo a algunos de ellos. El 69\% de los trabajadores encuestados afirman que son escuchados y se les permite colaborar en las actividades de la empresa, y el $31 \%$ señala que no son escuchados y no se les permite colaborar, siendo los directivos los que siempre toman las decisiones sin solicitar el parecer de los colaboradores.

El 58\% de los trabajadores encuestados afirman que tienen confianza en los compañeros con los cuales trabajan; por el contrario un $42 \%$ dice desconfiar de sus compañeros. El 78\% de los encuestados manifiesta que los directivos de la empresa buscan retener a los colaboradores con experiencia técnica y un $22 \%$ señala que no muestran interés en retener talento humano.

\section{Desarrollo personal}

En la tabla 3 de la página siguiente, se observa que, el 76\% de los encuestados dice estar cómodo con el ambiente físico en que labora y el $24 \%$ que no está conforme con el ambiente físico en el cual labora. El 71\% de los trabajadores encuestados manifiesta que los directivos muestran flexibilidad para pedir permiso y faltar al trabajo, y un $29 \%$ señala que no hay flexibilidad para permisos y faltas. El $70 \%$ de los encuestados dice que en sus empresas hay equidad en los salarios, y un $30 \%$ percibe como injustos los niveles salariales que reciben; y el $62 \%$ de los encuestados manifiesta que desearían que sus contratos fueran mejorados y el 38\% dice que están conformes con sus contratos. 
Tabla 2. Trabajo en equipo.

\begin{tabular}{lc}
\hline Pregunta & Resultado \\
\cline { 2 - 2 } & Q $\%$ \\
\hline
\end{tabular}

4) Solución de problemas

a) En nuestro trabajo generamos entre todos alternativas para la resolución de 20167 problemas

b) En nuestro trabajo se generan alternativas $99 \quad 33$ para la resolución de problemas

$300 \quad 100$

5) Escucha y colaboración

a) En el trabajo estamos acostumbrados a $207 \quad 69$ escuchar y colaborar.

b) En el trabajo no estamos acostumbrados a $93 \quad 31$ escuchar y colaborar. Total

$300 \quad 100$

6) Confianza entre compañeros a) Los trabajadores confiamos en nuestros $174 \quad 58$
compañeros.

b) Los trabajadores desconfiamos en nuestros compañeros.

$126 \quad 42$

Total $300 \quad 100$

7) Retención de personas con experiencia $\begin{array}{lll}\text { a) Para mi empresa es importante retener } & 234 & 78 \\ \text { personas con experiencias técnicas. } & \end{array}$

b) Para mi empresa es poco importante retener $66 \quad 22$ personas con experiencias técnicas.

$$
\text { Total }
$$

$300 \quad 100$

Fuente: Elaboración propia.

Tabla 3. Desarrollo personal.

\begin{tabular}{|c|c|c|}
\hline \multirow{2}{*}{ Pregunta } & \multicolumn{2}{|c|}{ Resultado } \\
\hline & $\mathrm{Q}$ & $\%$ \\
\hline \multicolumn{3}{|l|}{ 8) Ambiente físico } \\
\hline $\begin{array}{l}\text { a)En nuestra empresa existe flexibilidad para } \\
\text { pedir permiso y faltar a trabajar. }\end{array}$ & 213 & 71 \\
\hline $\begin{array}{l}\text { b)En nuestra empresa jamás existe flexibilidad } \\
\text { para pedir permiso y faltar a trabajar. }\end{array}$ & 87 & 29 \\
\hline Total & 300 & 100 \\
\hline \multicolumn{3}{|l|}{ 9) Permiso para faltar al trabajo } \\
\hline $\begin{array}{l}\text { a) En nuestra empresa existe flexibilidad para } \\
\text { pedir permiso y faltar a trabajar. }\end{array}$ & 213 & 71 \\
\hline $\begin{array}{l}\text { b)En nuestra empresa jamá } \\
\text { para pedir permiso y falt }\end{array}$ & 87 & 29 \\
\hline Total & 300 & 100 \\
\hline \multicolumn{3}{|l|}{ 10) Equidad en los salarios } \\
\hline $\begin{array}{l}\text { a)En nuestro trabajo existe equidad en los } \\
\text { salarios. }\end{array}$ & 210 & 70 \\
\hline $\begin{array}{l}\text { b)En nuestro trabajo no existe equidad en los } \\
\text { salarios. }\end{array}$ & 90 & 30 \\
\hline Total & 300 & 100 \\
\hline \multicolumn{3}{|l|}{ 11)Contratos } \\
\hline $\begin{array}{l}\text { a) En mi empresa desearíamos la mejora de } \\
\text { contratos. }\end{array}$ & 18 & 62 \\
\hline $\begin{array}{l}\text { b)En mi empresa estamos conformes con } \\
\text { nuestros contratos. }\end{array}$ & 114 & 38 \\
\hline Total & 300 & 100 \\
\hline
\end{tabular}

Fuente: Elaboración propia

\section{Adaptación al cambio.}

En la tabla 4 se observa que, , el $81 \%$ de los trabajadores encuestados manifiesta que no tienen problemas al hacer críticas constructivas sobre la empresa las cuales son aceptadas por los directivos, y un $19 \%$ señala que no pueden realizar críticas sobre la empresa. Un 76\% de los encuestados manifiestan que apoyan los planes de mejoramiento que se implementan en las empresas en las que laboran; un $24 \%$ cuestionan los planes de mejora que realizan en las empresas.

Tabla 4. Adaptación al cambio.

\begin{tabular}{|c|c|c|}
\hline Preounta & $\underline{\text { Resul }}$ & tado \\
\hline Pregunta & $\mathrm{Q}$ & $\%$ \\
\hline 12) Crítica constructiva & & \\
\hline $\begin{array}{l}\text { Nuestra empresa apoya la crítica } \\
\text { constructiva. }\end{array}$ & 243 & 81 \\
\hline $\begin{array}{l}\text { b) Nuestra empresa desaprueba la crítica } \\
\text { constructiva }\end{array}$ & 57 & 19 \\
\hline Total & 300 & 100 \\
\hline 13) Apoyo a planes de mejoramiento & & \\
\hline $\begin{array}{l}\text { a) Los empleados apoyamos los planes de } \\
\text { mejoramiento. }\end{array}$ & 243 & 76 \\
\hline $\begin{array}{l}\text { b) Los empleados cuestionamos los planes de } \\
\text { mejoramiento. }\end{array}$ & 57 & 24 \\
\hline Total & 300 & 100 \\
\hline utinas de trabajo & & \\
\hline $\begin{array}{l}\text { a) En mi empresa nunca se modifican las } \\
\text { rutinas de trabajo }\end{array}$ & 135 & 45 \\
\hline $\begin{array}{l}\text { b) En mi empresa se pueden modificar las } \\
\text { rutinas de trabajo. }\end{array}$ & 165 & 55 \\
\hline Total & 300 & 100 \\
\hline 15) $\mathrm{Cre}$ & & \\
\hline $\begin{array}{l}\text { a) Nuestra empresa impulsa constantemente el } \\
\text { crecimiento de todos. }\end{array}$ & 225 & 75 \\
\hline $\begin{array}{l}\text { b) Nuestra empresa raramente impulsa el } \\
\text { crecimiento de todos. }\end{array}$ & 75 & 25 \\
\hline Total & 300 & 100 \\
\hline
\end{tabular}

Fuente: Elaboración propia

Asimismo, se observa que, el $45 \%$ de los encuestados dice que no hay modificaciones en las rutinas de trabajo, pero el $55 \%$ manifiesta que si hay posibilidades de modificar las rutinas de trabajo.

Por otro lado, el $75 \%$ de los encuestados manifiestan que las empresas impulsan el crecimiento de todos sus colaboradores, en tanto que el $25 \%$ dicen que pocas veces se impulsa el crecimiento de un colaborador de las empresas.

\section{Mejora de la calidad y la productividad}

En la tabla 5 siguiente, se observa que, un $81 \%$ de los trabajadores encuestados dicen que son tan importantes los clientes internos como los externos, contrariamente un $19 \%$ manifiesta que no lo son. 
Tabla 5. Mejora de la calidad y la productividad.

\begin{tabular}{|c|c|c|}
\hline \multirow{2}{*}{ Pregunta } & \multicolumn{2}{|c|}{ Resultado } \\
\hline & $\bar{Q}$ & $\%$ \\
\hline \multicolumn{3}{|l|}{ 16) Clientes internos y externos } \\
\hline $\begin{array}{l}\text { a)Para nuestra organización son tan } \\
\text { importantes los clientes internos como los } \\
\text { externos. }\end{array}$ & 243 & 81 \\
\hline $\begin{array}{l}\text { b) Para nuestra organización son menos } \\
\text { importantes los clientes internos que los } \\
\text { externos. }\end{array}$ & 57 & 19 \\
\hline Total & 300 & 100 \\
\hline \multicolumn{3}{|l|}{ 17) Decisiones sobre alternativas } \\
\hline $\begin{array}{l}\text { a)Mi empresa me da la opción de poder } \\
\text { decidir y actuar en la búsqueda de } \\
\text { alternativas para resolver problemas }\end{array}$ & 225 & 75 \\
\hline $\begin{array}{l}\text { b)Mi empresa me limita la opción de } \\
\text { poder decidir y actuar en la búsqueda de } \\
\text { alternativas para resolver problemas }\end{array}$ & 75 & 25 \\
\hline Total & 300 & 100 \\
\hline
\end{tabular}

\section{8) Maquinaria de primera}

a)En el trabajo siempre se obtiene

maquinaria y equipo de primera para $174 \quad 58$

ofrecer servicios de bajo costo.

b)En el trabajo raramente se obtiene

maquinaria y equipo de primera para $126 \quad 42$

ofrecer servicios de bajo costo.

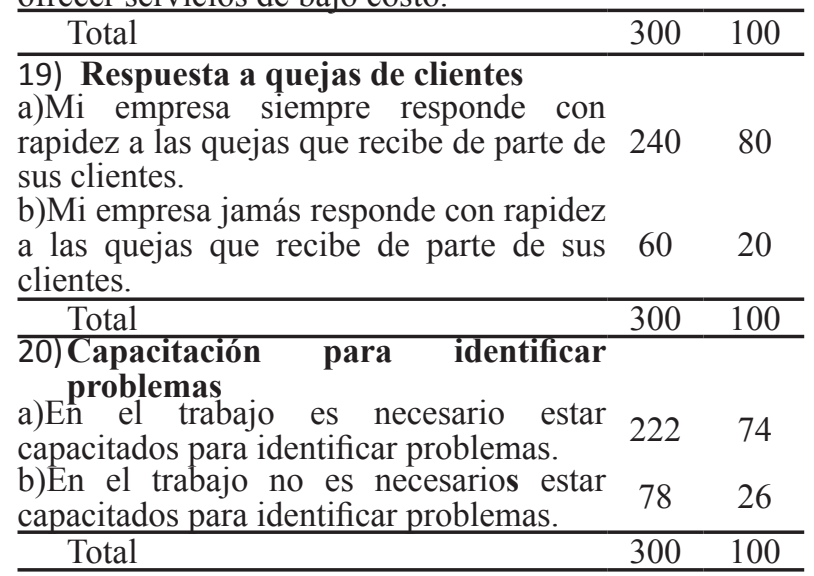

\section{1) Seguir criterio propio}

a)En el trabajo actuamos en función de $195 \quad 65$
nustro criterio propio

$105 \quad 35$

b)En el trabajo no actuamos en función de $105 \quad 35$

\begin{tabular}{lll}
\hline Total & 300 & 100 \\
\hline 22$)$
\end{tabular}

22) Mejora del servicio al cliente

$\begin{array}{lll}\text { a)En mi empresa se debe que mejorar } & 174 & 58 \\ \text { continuamente el servicio al cliente. } & \end{array}$

b)En mi empresa no se hace necesario

mejorar continuamente el servicio al $126 \quad 42$ cliente.

\begin{tabular}{ccc}
\hline Total & 300 & 100 \\
\hline
\end{tabular}

Fuente: Elaboración propia

Tabla 6. Propuesta de mejora de la cultura organizacional.

\begin{tabular}{|c|c|}
\hline Variable & $\begin{array}{c}\text { Acciones de mejora de la cultura } \\
\text { organizacional }\end{array}$ \\
\hline Comunicación & $\begin{array}{l}\text { - Comunicarse de manera clara y } \\
\text { directa, manteniendo el interés y } \\
\text { siendo honestos. } \\
\text { - Ser abiertos y directos al momento de } \\
\text { hacer preguntas. } \\
\text { - Dar personalmente las malas } \\
\text { noticias y escuchar y usar una forma } \\
\text { inteligente de pedir ayuda. } \\
\text { - Usar un lenguaje no verbal apropiado } \\
\text { - Com ser siempre positivo. } \\
\text { Comunicar las expectativas y pensar } \\
\text { antes de hablar. }\end{array}$ \\
\hline & $\begin{array}{l}\text { ara que } \\
\text { abajo en }\end{array}$ \\
\hline
\end{tabular}
equipo.

- Recompensar y reconocer el trabajo en equipo.

- Identificar el valor de una cultura de trabajo en equipo entre los miembros de la organización.

Trabajo en

equipo

- Diseñar objetivos alcanzables, motivantes y consensuados que dependen tanto del rendimiento individual y del equipo.

- Gestionar el rendimiento y la valoración del personal poniendo énfasis en el trabajo en equipo.

- Celebrar los éxitos del equipo en público.

- Tener claridad de las habilidades que se requieren en los colaboradores en función del mercado $\mathrm{y}$, por sobre todo, en función a las necesidades y estrategias del negocio.

- Ejercer un liderazgo que siempre tenga consideración y una búsqueda constante de talentos.

Desarrollo personal

- Establecer desafíos y metas claras, que sean una motivación constante entre los colaboradores de la empresa.

- Entrenar y preparar constantemente a los colaboradores en las últimas tecnologías y técnicas de sus distintas áreas, desarrollando sus capacidades $\mathrm{y}$ encontrando nuevos talentos que contribuyan al éxito de la empresa.

- Identificar situaciones y momentos que requieren de nuevas respuestas, adaptándose con eficacia a las distintas y variadas situaciones que se presentan en la convivencia y gestión con personas o grupos diversos.

Adaptación al cambio
- Responder constructivamente a los cambios en la organización y en su entorno personal.

- Aceptar los cambios y buscar las posibilidades y oportunidades de desarrollarse personal y profesionalmente. 
Continuación Tabla 6

\begin{tabular}{|c|c|}
\hline $\begin{array}{l}\text { Adaptación al } \\
\text { cambio }\end{array}$ & $\begin{array}{l}\text { - Apoya y promueve nuevos planes y } \\
\text { procesos, encontrando y/o elaborando } \\
\text { soluciones nuevas para mejorar las } \\
\text { estrategias existentes de la empresa. } \\
\text { - Abordar la incertidumbre y, al mismo } \\
\text { tiempo, conserva la confianza y } \\
\text { el entusiasmo por conseguir los } \\
\text { objetivos. }\end{array}$ \\
\hline $\begin{array}{l}\text { Mejora de la } \\
\text { calidad } \\
\text { y } \\
\text { productividad }\end{array}$ & $\begin{array}{l}\text { - Proporcionar, de forma consistente } \\
\text { una calidad de servicio superior al } \\
\text { de la competencia. } \\
\text { Desarrollar una oferta, una } \\
\text { entrega y una imagen de servicios } \\
\text { diferenciada. } \\
\text { - Diseñar un servicio más efectivo, } \\
\text { reduciendo la necesidad de ciertos } \\
\text { servicios y presentando incentivos } \\
\text { a los clientes para que presten } \\
\text { servicios a la empresa. } \\
\text { - Hacer que los suministradores de los } \\
\text { servicios trabajen más duramente } \\
\text { y/o con más capacidad. } \\
\text { Alcanzar y sostener una alta } \\
\text { productividad, medida contra sus } \\
\text { mejores rivales. } \\
\text { Mantener un proceso de mejora } \\
\text { repetido cotidianamente que } \\
\text { permita ir mejorando la calidad de } \\
\text { los servicios, la productividad, las } \\
\text { relaciones con nuestros clientes y el } \\
\text { margen de ganancias. }\end{array}$ \\
\hline
\end{tabular}

Fuente: Elaboración propia

El $75 \%$ de los encuestados dicen que en las empresas en que laboran tienen la oportunidad de participar en la solución de problemas y el $25 \%$ que su participación en la resolución de problemas es muy limitada.

En la tabla 5 también se observa que el $80 \%$ de los encuestados dicen que en las empresas en que laboran se responden siempre con rapidez a las quejas de los clientes el 20\% que no se responden con la prontitud que el cliente quisiera. El $74 \%$ de los trabajadores está consciente de que deben estar capacitados para resolver los problemas que surgen en el área en que laboran en las empresas, un $26 \%$ opina que no es necesario capacitación para hacerlo. El $58 \%$ de los encuestados dicen que se debe mejorar el servicio para satisfacción de los clientes, mientras que un $42 \%$ considera que no es necesario hacerlo.

\section{Acciones de mejora de la cultura organizacional.}

Laculturaorganizacionalesunodelospilaresfundamentales para apoyar a todas aquellas organizaciones que quieren hacerse competitivas. En tal sentido, las acciones de mejora que se plantean en la tabla se revisten de gran importancia, puesto que obedecen a la necesidad de mejorar los aspectos deficientes en la cultura organizacional de las empresas de servicios de Lima.

\section{Discusión}

El diagnóstico eficaz de la cultura organizacional constituye un factor importante en toda empresa para mantenerse vigente en el contexto en el que se encuentra inmersa pues la cultura organizacional se manifiesta a través del comportamiento de los colaboradores en las empresas. Los resultados de la primera variable de la cultura en las empresas de servicios estudiada: Comunicación se muestran en la Fig. 1 siguiente.

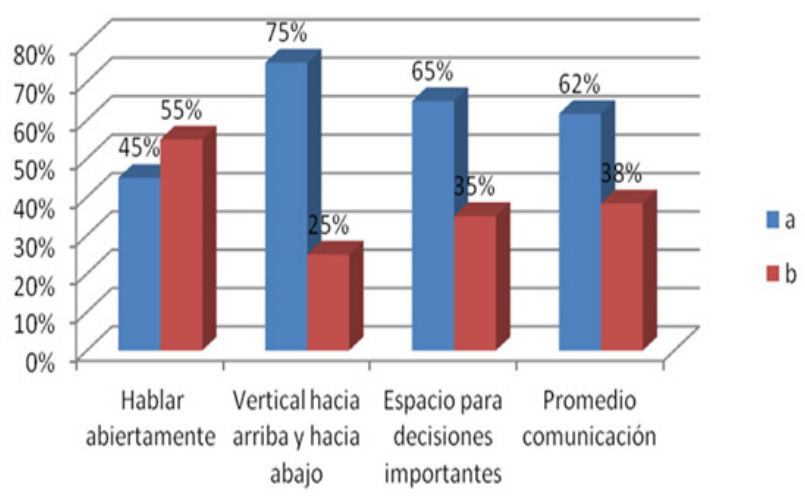

Figura 1. Comunicación.

Fuente: Elaboración propia

El promedio de eficacia de la comunicación es del $62 \%$. Al respecto, Álvarez (2011) señala que la cultura es el marco de referencia fundamental para interpretar la realidad, y que al proceso de hacer a la cultura consciente y plenamente operativa contribuye de modo fundamental la comunicación. (8). A su vez, Andrade (2005) desarrollar una cultura única se logra definiendo con claridad sus valores y las conductas que deben derivarse de ellos, difundiéndolas ampliamente y estableciendo los mecanismos que garanticen el cumplimiento de estas pautas conductuales. (9). En tal sentido, Morales

(2012), indica que las bases de una cultura organizacional es la constante comunicación de los valores principales de la empresa, entendiéndose que el hecho de tener un set de valores es para que los directivos, empleados y clientes sepan de qué se trata la organización y cómo funciona. Asimismo, si bien la tecnología ha ayudado al proporcionar avances en cuestiones de comunicación, en una organización siempre será crítico el poder comunicar las cosas cara a cara, porque de esa manera se asegura de varias cosas muy importantes al momento de comunicar: el mensaje fue recibido, el mensaje fue entendido, el mensaje fue claro, el mensaje cumplió su propósito. Por consiguiente, trabajar desde la comunicación para transmitir, mantener y fomentar la cultura organizacional implica que esta debe estar bien consolidada y debe tener poder para hacer las cosas, donde la alta gerencia la respeta como un área estratégica para el desarrollo organizativo.

El trabajo en equipo fue la segunda variable estudiada. Los resultados consolidados para esta variable se muestran en la Fig. 2 a continuación. 


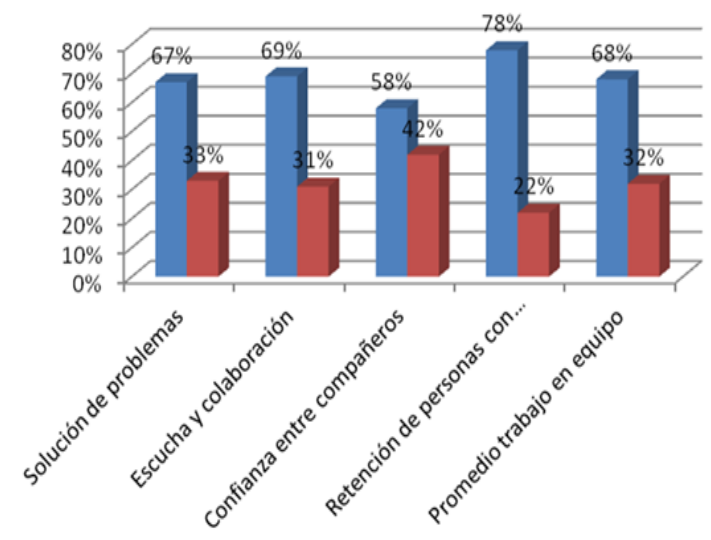

Figura 2. Trabajo en equipo.

Fuente: Elaboración propia

El promedio de eficacia del trabajo en equipo es del $68 \%$. La cultura organizativa afecta las conductas de sus empleados, ellos necesitan cumplir con el rol esperado por la empresa. El sentimiento de pertenencia, hace que los individuos, readapten sus valores y quieran corresponder con lo requerido. Sobre esto, Mora (2010), señala que es muy importante entender que la gerencia encargada de dirigir la empresa esté plenamente identificada con lo que representa la cultura organizacional, el cómo esta se ha venido desarrollando, su rol, alcance, repercusiones y de qué manera ella ha incidido en el comportamiento y logros de los equipos de trabajo. En las organizaciones, el trabajo en equipo no solo produce mejoras individuales y organizacionales, sino que interviene también en el perfeccionamiento de los servicios, tanto cualitativa como cuantitativamente y facilita una mejor gestión de la información y el conocimiento. El trabajo en equipo es parte de la nueva consciencia de las organizaciones modernas, pues es un hecho que cuando todos comparten las metas de una organización, los resultados son altamente satisfactorios. Fomentar el trabajo en equipo es la creación de una cultura de trabajo en la que prime el valor de la colaboración. En un entorno de trabajo en equipo, la gente entiende y cree que el pensamiento, la planificación, las decisiones y acciones son mejores cuando se hacen en forma cooperativa. La gente reconoce, e incluso asimila, la creencia de que "ninguno de nosotros es tan bueno como todos nosotros." Por ejemplo, tomando como base los factores culturales anteriores para su análisis en organizaciones estadounidenses y japonesas, los investigadores Nahavandi y Aranda (1994), llegan a concluir que los valores culturales japoneses permiten la formación de equipos en las organizaciones, mientras que los valores culturales de las organizaciones estadounidenses no contribuyen a que los esfuerzos en la formación de equipos sean exitosos. De allí que el Trabajo en Equipo resulta una actividad desafiante e integradora que además puede involucrar a individuos de diversas áreas y niveles de la organización o aún de su entorno.

El desarrollo personal fue la tercera variable estudiada. Los resultados consolidados de los reactivos utilizados se aprecian en la Fig. 3 siguiente. 174

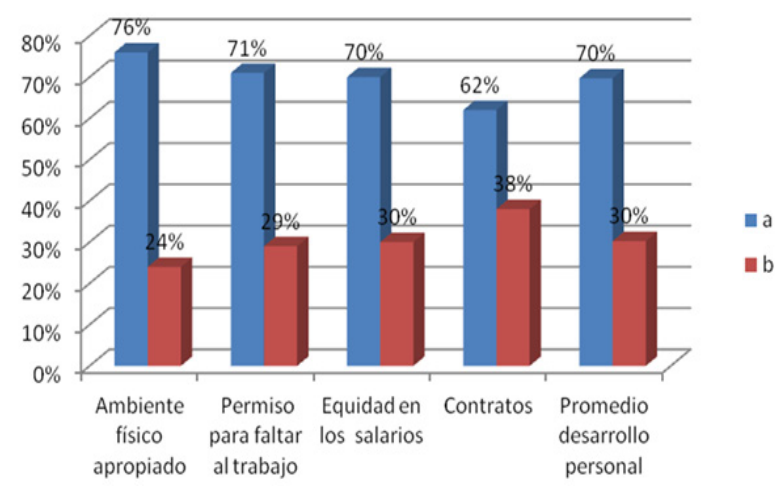

Figura 3. Desarrollo personal.

Fuente: Elaboración propia

El promedio de eficacia del desarrollo personal es del $70 \%$. La cultura organizacional sirve de marco de referencia a los miembros de la organización y da las pautas acerca de cómo las personas deben conducirse en ésta. La necesidad de desarrollar cultura organizacional efectiva implica como un factor importante el desarrollo de las personas. En este sentido, la formación es el instrumento fundamental para que las personas mejoren aptitudes y actitudes en el trabajo, y es esencial en los procesos de cambio. Al mismo tiempo, el desarrollo personal y profesional de las personas, que es necesario para estar intelectualmente vivas, requiere de formación permanente. La formación debe abarcar todos los niveles de la empresa. La formación en habilidades directivas a todo el personal con mando es vital, como lo es la formación de los trabajadores en las actividades preventivas en las que debe estar prevista su participación. La organización debería estar en continuo aprendizaje. (Ministerio de Trabajo y Asuntos Sociales, España, 2012). La idea de realizar esta actividad es lograr que la persona sea un ser más completo e íntegro. Al alcanzar esta estabilidad emocional, entonces el individuo podrá disfrutar de un mayor bienestar personal y laboral; podrá mejorar su potencial, excelencia, responsabilidad, autoestima y creatividad en su ambiente de trabajo. (Challa, 1992). Es por ello que Zapata (2001), manifiesta que el ser competitivo no es un atributo independiente del personal, si una organización es competitiva será porque su personal es competitivo. La condición de la empresa es consecuencia de las condiciones de los recursos principalmente el recurso humano.

La adaptación al cambio fue la cuarta variable estudiada. Los resultados consolidados para esta variable se muestran en la Figura 4 que se presenta a continuación.

El promedio de eficacia de la adaptación al cambio es del $69 \%$. El nuevo escenario a que están sujetas las organizaciones, son los cambios apresurados que demandan alta flexibilidad y capacidad de adaptación a las exigencias de su entorno. En este sentido, deben entenderse los cambios como retos permanentes capaces de asegurar el fracaso o el éxito de una organización. En la 
medida que los cambios se vuelven un factor permanente y acelerado, la adaptabilidad del individuo organizacional a tales cambios resulta cada vez más determinante en la supervivencia de cualquier empresa.

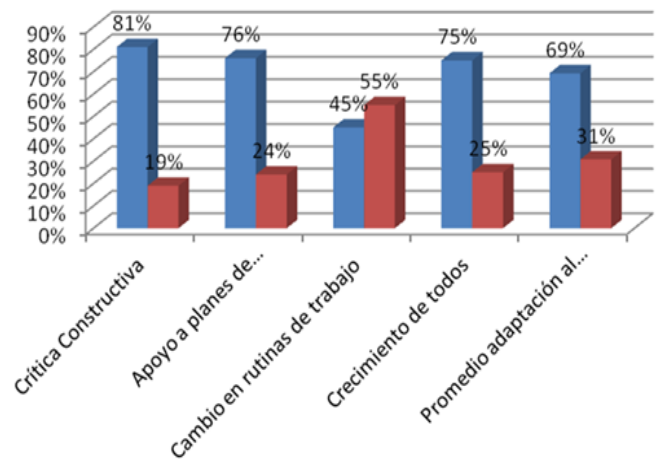

Figura 4. Adaptación al cambio.

Fuente: Elaboración propia

En tal sentido, Ramírez (2007) señala que, la misma supervivencia de una organización puede depender de la forma como adapte la cultura a un ambiente de rápido cambio; cambiar las organizaciones requiere cambiar su cultura. De este modo, Scott y Jaffe en su texto sobre "Como Dirigir el Cambio en las Organizaciones", manifiestan que el proceso de hacer un cambio de importancia en la cultura de las empresas, exige que las personas se olviden de "cómo eran las cosas" y enfrenten un periodo de duda e incertidumbre y que, las empresas que utilizan bien el proceso de modificar la cultura de la organización, reducen el tiempo requerido para hacer cambios similares en el futuro.

La mejora de la calidad y la productividad fue la quinta variable estudiada. Las empresas de servicios reciben fuertes presiones para mejorar su calidad y productividad, puesto que al tener mucho personal, los costes crecen rápidamente. La investigación en gestión de calidad dentro del sector servicios no está todavía tan desarrollada como lo está para el caso de empresas manufactureras.

Los resultados consolidados para esta variable se muestran en la Fig. 5 en la página siguiente.

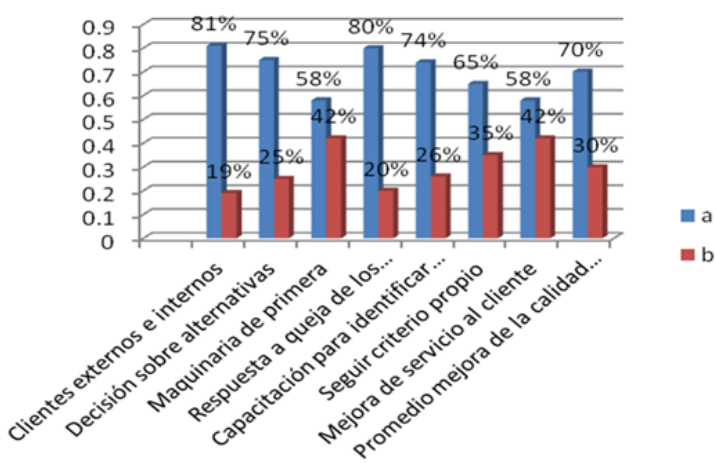

Gráfico 5. Mejora de la calidad y productividad. Fuente: Elaboración propia
En un trabajo de naturaleza teórica, Lemak \& Reed (2000) postulan que el éxito de un TQM en las empresas de servicios descansa sobre la adecuación entre la interdependencia y el contenido del TQM, entendido como integrado por los cuatro componentes principales, y los procesos, específicamente liderazgo, formación, equipos y cultura. La productividad de una organización de servicios depende de dos factores: las características del servicio que se brinda, y la eficiencia para producirlo. Para alcanzar una ventaja competitiva sobre los rivales, pueden elegirse dos caminos: buscar una ventaja en los costos de producción, o alcanzar en el servicio una diferenciación valiosa para los clientes. Entonces, las ventajas competitivas de cualquier organización se mantienen mejorando continuamente su productividad. Una empresa de servicios competitiva es, entonces, la que puede alcanzar y sostener una alta productividad, medida contra sus mejores rivales. (FRIGO, 2012).

\section{Conclusiones}

En base al estudio realizado se observó que en las empresas de servicios de Lima metropolitana estudiadas la eficacia de la cultura organizacional está relacionada con: la comunicación, trabajo en equipo, desarrollo personal, adaptación al cambio y mejora de la calidad y productividad y alcanza un nivel promedio de eficacia del $67.8 \%$. La comunicación en las empresas de servicios presenta una eficacia del $62 \%$ frente a un nivel de ineficacia del $38 \%$, lo cual denota en gran medida que en ellas se desarrolla relaciones de trabajo que se dan en un ambiente de sinceridad, de escuchar al otro y de circulación de la información, impulsando el conocimiento, la calidad, la productividad, la capacidad de resolución de problemas, corresponsabilidad y el compromiso necesario para alcanzar objetivos estratégicos en la organización. El trabajo en equipo alcanza una eficacia del $68 \%$ frente a un nivel de ineficacia del $32 \%$, resultado importante expresado fundamentalmente en la integración de las personas por el trabajo grupal, el incremento productivo como consecuencia de las ganancias en motivación que participan en los equipos de trabajo en las empresas de servicios estudiadas. El desarrollo personal presenta un nivel de eficacia del $70 \%$ frente a una ineficacia del $30 \%$, lo cual pone de manifiesto los esfuerzos de las organizaciones por capacitar y desarrollar a sus colaboradores para desempeñar su trabajo actual y en aspectos del ser que facilitan o permiten la expansión de sus competencias necesarias para cumplir con sus responsabilidades y desafíos futuros. La adaptación al cambio alcanza una eficacia del $69 \%$ frente a una ineficacia del $31 \%$, lo cual pone de manifiesto los esfuerzos de las organizaciones por capacitar y desarrollar a sus colaboradores para desempeñar su trabajo actual y en aspectos del ser que facilitan o permiten la expansión de sus competencias necesarias para cumplir con sus responsabilidades y desafíos futuros. La mejora de la calidad y productividad adaptación al cambio alcanza una eficacia del $70 \%$ frente 
a una ineficacia del 30\%, nivel de eficacia que se debe principalmente a la rápida respuesta a las quejas de los clientes y la identificación de problemas en un tiempo adecuado asegurando una solución de los mismos.

\section{Literatura citada}

Aguilar, A. 2009. El diagnóstico de la cultura organizacional o la cultura de las culturas. México. Instituto Tecnológico y de estudios Superiores de Monterrey En Global media Journal Edición Iberoamericana, Volúmen 6, Número 11 p. 67-81.

Álvarez, C. 2011. Cultura organizacionaly comunicación interna. Contenidos Infosalarial

http://www.infosalarial.com/contenidos/index. php?option=com_content $\&$ view $=$ article $\&$ id $=2643$ : cult ura-organizacional-y-comunicacioninterna\&catid $=125$ : profesionales\&Itemid=54 Accesado: 06/07/12

Andrade, H. 2005. Comunicación organizacional interna: proceso, disciplina y técnica. $1^{\text {a }}$ Edición. Netbiblo. S.L. p. 71.

Challa, B. 1992. Relaciones humanas. Pág. 112. Citado por CALANCHE S. et.al. (2011) ¿Cómo influye la capacitación y el desarrollo personal en el éxito de la organización? Santa Marta. Corporación Unificada Nacional de educación Superior.

http://talentohumanocun.wikispaces.com/file/view/2 Doc_CAPACITACION+Y+DESARROLLO.pdf. p. 2 .

Cruz, T. 2005. El diagnóstico de la cultura empresarial. Experiencia de una empresa. Gestiópolis. http://www. gestiopolis.com/canales5/ger/diaorgaex.htm.

Fernández, D. 2010. 5 problemas de las pymes para el éxito. México. CNN Expansión. p.1.

h t t p : / / w w w . c n n ex pan s i o n . c o m / emprendedores/2010/07/21/ser-exitoso-sin-morir-en-elintento.

Frigo, E. 2012. ¿Qué es la competitividad?, ¿cómo se la consigue? y después de conseguida, ¿cómo se la mantiene? Foro de Seguridad http://www.forodeseguridad.com/ artic/admin/adm_5115.htm. Accesado: 11/14/12

Lemak, D. J. \& Reed, R. 2000. «An application of Thompson's typology to TQM in service firms», Journal of Quality Management, Vol.5, pp.67-83.

Ministerio de Trabajo y Asuntos Sociales España. 2012. NTP 745: Nueva cultura de empresa y condiciones de trabajo.

http://www.insht.es/InshtWeb/Contenidos/ Documentacion/FichasTecnicas/NTP/Ficheros/701a750/ ntp_745.pdf

Mora, C. 2010. Cultura organizacional y equipos de trabajo. Tópicos gerenciales modernos.

http://topicosgerencialesmodernos.lacoctelera.net/ post/2010/05/01/cultura-organizacional-y-equipostrabajo
Morales, O. 2012. El core de la cultura organizacional. http://oscarimorales.com/2012/06/el-core-de-la-culturaorganizacional/

Pérez L., M. L. 1998. Desarrollo de un instrumento de medición que permite medir la cultura organizacional. Santa Catarina Mártir, Puebla; citado por PABLO PABLO, MARILYN y SALDAÑA, M. 2005. Análisis de la cultura organizacional, evaluación de la imagen e identidad corporativa para la creación de manuales corporativos para la empresa transportes Saldaña Granda S.A. de C.V. Puebla. Universidad de las Américas Puebla. Escuela de Ciencias Sociales. Departamento de Ciencias de la Comunicación. http://catarina.udlap. mx/u_dl_a/tales/documentos/lco/pablo_a_m/capitulo5. pdf

Ramírez, C. 2007. Proceso de cambio en una empresa. Monografías .com. http://www.monografias.com/ trabajos13/prodcam/prodcam.shtml. p.1.

Schein, E. 1988. La cultura empresarial y el liderazgo, una visión dinámica. Barcelona. Plaza \& Janés. p. 25-26. http://www.insht.es/InshtWeb/Contenidos/ Documentacion/FichasTecnicas/NTP/Ficheros/701a750/ ntp_745.pdf

Scott, C. D. y Jaffe, D. T. 1993. Como dirigir el cambio en las organizaciones. México. Grupo editorial Iberoamérica, citado por GARCÍA, VERÓNICA. 2002. El cambio organizacional.

http://www.ucapanama.org/ovasdo/cambio_ organizacional.pdf

Teamwork. Fomentar el trabajo en equipo en una organización. http://www.eltrabajoenequipo.com/ Organizaciones.htm Accesado: 07/15/12.

Vargas, J. G. 2007. La cultura organizacional en México. Edición electrónica gratuita. www.eumed.net/ libros/2007b/ 301. p.

Zapata, G. 2001. Desarrollo del personal: ventaja competitiva en la empresa. Gestiópolis.

http://www.gestiopolis.com/canales/derrhh/articulos/ no5/personalgunnar.htm 